the reduction (due to behaviour change only) would be $\sim 50 \%$, from $8-12$ to $5-7$. If the duration of $\mathrm{PHI}$ is shorter, or testing frequency is lower frequency, and/or viralload suppression is slower then the number of infections averted is reduced.

Conclusion Diagnosing HIV during PHI can markedly reduce transmission because its high infectivity and short duration make treatment and even short-term behaviour change effective in reducing transmission. Cost-effectiveness would be increased by efficient approaches to identifying PHI (e.g. encouraging HIV testing after episodes of high-risk behaviour and intensive contact tracing from recently-infected individuals, since PHI cases are likely to be clustered). Our quantification of the number of infections averted is an essential component of assessment of the cost-effectiveness of strategies to increase diagnoses of PHI.

\section{P5.24 DRUG RESISTANCE AMONG WOMEN ATTENDING ANTENATAL CLINIC IN GHANA}

Philip Enyan. University of Ghana, Ghana

10.1136/sextrans-2017-053264.640

Introduction Initial evidence from resource-limited countries using the WHO HIV drugresistance (HIVDR) threshold survey suggests that transmission of drug-resistance strains islikely to be limited. However, as access to ART is expanded, increased emergence of HIVDR isfeared as a potential consequence. We have performed a surveillance survey of transmittedHIVDR among recently infected persons in the geographic setting of Accra, Ghana.

Methods As part of a cross-sectional survey, 2 large voluntary counselling and testing centres inAccra enrolled 50 newly HIV-diagnosed, antiretroviral drug-naïve adults aged 18 to 25 years.Virus from plasma samples with $>1,000$ HIV RNA copies/mL (Roche Amplicor v1.5) weresequenced in the pol gene. Transmitted drug resistance-associated mutations (TDRM) wereidentified according to the WHO 2009 Surveillance DRM list, using Stanford CPR tool (v 5.0beta). Phylogenetic relationships of the newly characterised viruses were estimated bycomparison with HIV-1 reference sequences from the Los Alamos database, by using theClustalW alignment program implemented.

Results Subtypes were predominantly D (39/70, 55.7\%), A $(29 / 70,41.4 \%)$, and C $(2 / 70 ; 2,9 \%)$. Seven nucleotide sequences harboured a major TDRM (3 NNRTI, 3 NRTI, and 1 PIassociated mutation); HIVDR point prevalence was $10.0 \%$ (95\%CI $4.1 \%$ to $19.5 \%)$. The identified TDRM were D67G (1.3\%), L210W (2.6\%); G190A (1.3\%); G190S (1.3\%); $\mathrm{K} 101 \mathrm{E}(1.3 \%)$, and N88D (1.3\%) for PI.

Conclusion In Accra the capital city of Ghana, we found a rate of transmitted HIVDR, which,according to the WHO threshold survey method, falls into the moderate $(5 \%$ to $15 \%)$ category.This is a considerable increase compared to the rate of among women attending an antenatal clinic in mamobi. As ART programs expand throughoutAfrica, incident infections should be monitored for the presence of transmitted drug resistance inorder to guide ART regimen policie.

\section{P5.25 FEASIBILITY OF A POINT-OF-CARE MODEL FOR THE PREVENTION OF MOTHER TO CHILD TRANSMISSION OF SYPHILIS IN CALI, COLOMBIA}

${ }^{1}$ Potes LI, ${ }^{1}$ Rubiano LC, ${ }^{1}$ Ramírez LG, ${ }^{2}$ Tunubalá GA, ${ }^{1}$ Orobio Y, ${ }^{3}$ Salazar JC. ${ }^{1}$ CIDEIM, Colombia; ${ }^{2}$ ESE Norte, Colombia; ${ }^{3}$ University of Connecticut School of Medicine and Connecticut Children's Medical Centre

\subsection{6/sextrans-2017-053264.641}

Introduction Most women in Colombia receive antenatal care (ANC), but goals for the reduction of congenital syphilis (CS) have not been met. In 2015 Cali had an incidence of 3.0 cases of CS per 1000 births.Point of care testing (POC) has proven cost-effective, and since 2015 it is now recommended by the Colombian Ministry of Health Guidelines. Nevertheless, POC for gestational syphilis (GS) has not been implemented in ANC. We sought to test the feasibility of POC and identify barriers to its implementation in Floralia, a public, primary healthcare centre in Cali.

Methods During a three-month period, we observed routine ANC in Floralia and offered women on-site rapid tests (RT) for syphilis. Process measures were compared with data from the clinical charts (CC) of women seen in 2015. Patients and administrators were interviewed and asked to describe ANC, syphilis screening procedures, and to identify opportunities for improvement.

Results 55 women were offered POC testing, 42 agreed to participate and were screened. 55 CC from 2016, and 123 CC from 2015, were reviewed. The median gestational age at which women sought health care was eight weeks. The first trimester of pregnancy had the highest percentage of women screened for GS: $97 \%$ were screened in 2015, and $100 \%$ were screened in 2016. Changes to screening procedures were hindered by existing contracts with insurance companies (IC); they determine what tests can be ordered and who can order them. Administrative procedures lead to missed opportunities for screening and treatment.Two women (1.6\%) from the 2015 group were diagnosed with GS, and three (5.4\%) were diagnosed during the intervention. POC testing allowed us to treat GS on the same day of the positive result, while using routine testing methods took 6 to 24 days.

Conclusion Diffusion of the national guidelines for GS has been insufficient to control GS and CS: There is still confusion among health care workers, and contracts with IC do not include RT. POC for syphilis can be a part of ANC, but barriers need to be addressed before implementation.

\section{P5.26 ATYPICAL PAP SMEAR BEFORE AND AFTER HPV VACCINE IN PRIVATE SERVICE AT FORTALEZA, BRAZIL}

${ }^{1}$ Renata Mirian Nunes Eleuterio, ${ }^{1} J o s e ́$ Eleutério Junior, ${ }^{1}$ Ana Larissa Duarte, ${ }^{2}$ Paulo Giraldo,

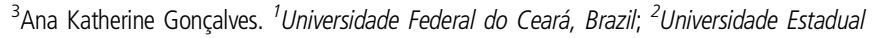
de Campinas - UNICAMP, Brazil; ${ }^{3}$ Universidade Federal do Rio Grande do Norte, Brazil

\subsection{6/sextrans-2017-053264.642}

Introduction HPV vaccine is a powerful tool in to prevent cervical cancer. It were commercially introduced in Brazil in 2008 and only in 2013 the government starts in public health.Objectives: To assess the influence of the introduction of HPV vaccine in Fortaleza (Brazil) in the results of Pap smear.

Methods The results of Pap smear between 2006 and 2014 in Prof. Eleutério Laboratory were searched in the files to 